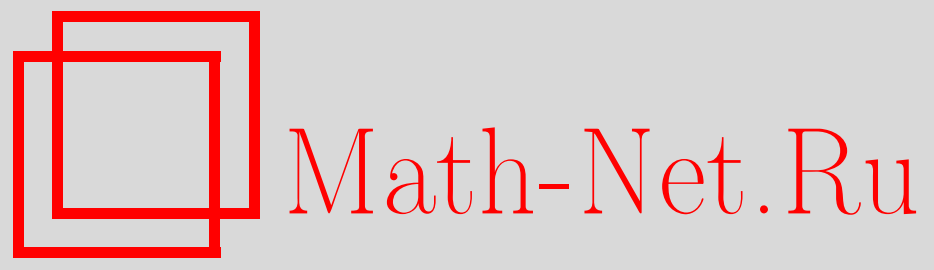

И. А. Ибрагимов, Об оценке многомерной регрессии, Теория вероятн. и ее примен., 2003, том 48, выпуск 2, 301-320

DOI: https://doi.org/10.4213/tvp286

Использование Общероссийского математического портала MathNet.Ru подразумевает, что вы прочитали и согласны с пользовательским соглашением

http: //www.mathnet.ru/rus/agreement

Параметры загрузки:

IP : 3.89 .197 .203

26 апреля 2023 г., $16: 22: 43$

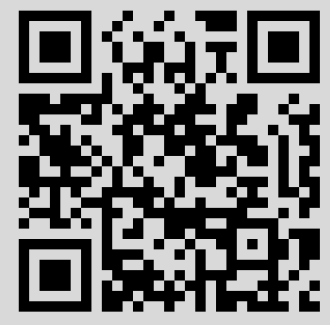


(C) 2003 г.

ИБРАГИМОВ И. А.*

\section{ОБ ОЦЕНКЕ МНОГОМЕРНОЙ РЕГРЕССИИ ${ }^{1)}$}

Пусть $(X, Y)$ - случайный вектор, первая компонента которого принимает значения в измеримом пространстве $(\mathfrak{X}, \mathfrak{A}, \mu)$ с мерой $\mu$, тогда как $Y$ - это вещественнозначная случайная величина. Пусть

$$
f(x)=\mathbf{E}\{Y \mid X=x\}
$$

- функция регрессии $Y$ на $X$. Мы рассматриваем задачу оценивания $f(x)$ по наблюдениям $n$ независимых копий вектора $(X, Y)$ в предположении, что $f \in \mathbf{F}$, где $\mathbf{F}-$ a priori известное множество с заданными метрическими характеристиками такими, как $\varepsilon$-энтропия или поперечники Колмогорова.

Ключевые слова и фразы: аддитивная регрессия, непараметрическое оценивание, регрессия, функция регрессии.

1. Введение. Основные результаты. Рассмотрим случайный вектор $(X, Y)$, первая компонента которого, $X$, принимает значения в измеримом пространстве $(\mathfrak{X}, \mathfrak{A}, \mu)$, тогда как вторая компонента, $Y$, это вещественнозначная случайная величина. Обозначим

$$
f(x)=\mathbf{E}\{Y \mid X=x\}
$$

функцию регрессии $Y$ на $X$. Ниже мы рассматриваем задачу оценивания функции $f(x)$ по $n$ независимым наблюдениям

$$
\left(\mathbf{X}_{n}, \mathbf{Y}_{n}\right)=\left(X_{1}, Y_{1}\right), \ldots,\left(X_{n}, Y_{n}\right)
$$

вектора $(X, Y)$. Если $\hat{f}$ - какая-нибудь оценка для $f$, то в качестве функции потерь мы выберем $\|f-\hat{f}\|^{2}$, где $\|\cdot\|,(\cdot, \cdot)$ означают норму и скалярное произведение в $L_{2}(\mathfrak{X})$ соответственно. При этом задача оценивания решается в предположении, что подлежащая оцениванию неизвестная функция $f \in \mathbf{F}$, где $\mathbf{F} \subset L_{2}(\mathfrak{X})$ - некоторое известное множество

* С.-Петербургское отделение Математического института им. В. А. Стеклова РАН, ул. Фонтанка, 27, 191011 С.-Петербург, Россия, математико-механический факультет СПбГУ; e-mail: ibr32@pdmi.ras.ru

1) Работа выполнена при частичной поддержке РФФИ (гранты 02-01-00262 и 00-15-96019) и РФФИ-DFG (грант 99-01-04027). 
функций. В этом случае качество оценки $\hat{f}$ можно измерять, соотнося его с величиной

$$
\Delta_{n}(\mathbf{F})=\inf _{\hat{f}} \sup _{f \in \mathbf{F}} \mathbf{E}_{f}\left\{\|\hat{f}-f\|^{2}\right\}
$$

Асимптотическое поведение $\Delta_{n}$ достаточно хорошо изучено, когда $\mathfrak{X}$ есть область в $\mathbf{R}^{d}$, а $\mathbf{F}$ - какой-нибудь класс гладких функций. Если, например, $\mathbf{F}$ включает в себя все функции $f\left(x_{1}, x_{2}, \ldots, x_{d}\right)$, имеющие по переменной $x_{j}, j=1,2, \ldots, d, r_{j}$ производных в $L_{2}$, причем последняя производная удовлетворяет в $L_{2}$ условию Гёльдера порядка $\alpha_{j}$ и $r_{j}+\alpha_{j}=\beta_{j}, j=1,2, \ldots, d$, то из [6]-[8], [14] легко следует, что $\Delta_{n} \geqslant c n^{-2 \beta /(2 \beta+1)}$, где $\beta$ определяется из соотношения $\beta^{-1}=$ $\beta_{1}^{-1}+\beta_{2}^{-1}+\cdots+\beta_{d}^{-1}$, а постоянная $c>0$. Таким образом, с ростом размерности $d$ величина ошибки оценивания быстро возрастает. В связи с этим в прикладных исследованиях предлагались различные регрессионные модели, в которых величина погрешности не возрастала бы столь заметно с ростом размерности (см. [3]). Одна из таких моделей, аддитивная схема регрессии, исследована в работе [15]. В этой работе предполагается, что $\mathfrak{X}$ - это единичный куб в $\mathbf{R}^{d}$, функция $f$ - сумма $d$ функций одного вещественного переменного,

$$
f\left(x_{1}, x_{2}, \ldots, x_{d}\right)=f_{1}\left(x_{1}\right)+f_{2}\left(x_{2}\right)+\cdots+f_{d}\left(x_{d}\right)
$$

и каждая из функций $f_{j}$ имеет гладкость $\beta$ в указанном выше смысле (но в метрике $C(\mathfrak{X})$, а не $\left.L_{2}(\mathfrak{X})\right)$. Автор этой работы, Ч. Стоун, показал, в частности, что при этих и некоторых дополнительных предположениях относительно распределения $X$ можно построить оценки $\hat{f}_{n}$, для которых при $n \rightarrow \infty$

$$
\mathbf{P}\left\{\mathbf{E}\left\{\left\|\hat{f}_{n}-f\right\|^{2} \mid X_{1}, X_{2}, \ldots, X_{n}\right\}=O\left(n^{-2 \beta /(2 \beta+1)}\right)\right\} \longrightarrow 1,
$$

и, таким образом, для аддитивных моделей порядок убывания погрешности уже не зависит от размерности.

Результат Стоуна, конечно, объясняется тем, что множество функций вида (1.1) существенно уступает в массивности множеству всех функций $d$ переменных гладкости $\beta$. Ниже мы предлагаем границы величины погрешности в сформулированной выше общей задаче регрессии в терминах метрических характеристик массивности множества $\mathbf{F}$ как подмножества $L_{2}(\mathfrak{X})$. В качестве таких характеристик используются поперечники по Колмогорову и Бернштейну и $\varepsilon$-энтропия.

$N$-поперечник по Колмогорову множества $\mathbf{F}$ в $L_{2}(\mathfrak{X})$ - это величина наилучшего приближения $\mathbf{F}$ линейными многообразиями размерности $\leqslant N$ :

$$
d_{N}(\mathbf{F})=\inf _{\left\{M_{N}, a\right\}} \sup _{x \in \mathbf{F}} \inf _{y \in M_{N}+a}\|x-y\|
$$


где $M_{N}$ пробегает все подпространства $L_{2}(\mathfrak{X})$ размерности $\leqslant N$. Этот поперечник был введен А.Н. Колмогоровым в 1936 г. в работе [10].

$N$-поперечник по Бернштейну множества $\mathbf{F}$ - это радиус максимального $N$-мерного диска, который можно вписать в $\mathbf{F}$ :

$$
b_{N}(\mathbf{F})=\sup _{\left\{M_{N}, a\right\}} \sup \left\{r: \mathbf{F} \cap M_{N} \supset B \cap\left(M_{N}+a\right)\right\} .
$$

Здесь $B$ означает единичный шар пространства $L_{2}$, а $M_{N}$ пробегает все $N$-мерные подпространства $L_{2}(\mathfrak{X})$.

Подробнее об этих и других поперечниках см. [2], [16], [17]. Отметим; что впервые поперечники при исследовании задач статистики применил Н.Н. Ченцов в работе [18]. Н.Н. Ченцов говорил, что он в свою очередь находился под влиянием доклада А.Н. Колмогорова, где тот указывал на возможную роль поперечников при исследовании некорректных задач. (О влиянии идей А. Н. Колмогорова на решение непараметрических задач оценивания см. также [9].)

3 а м е ч а н и е. Ниже для простоты мы предполагаем, не оговаривая этого особо, что все приближающие линейные многообразия $M_{N}+a$ являются подпространствами, т.е. что $a=0$.

Наложим на рассматриваемую задачу регрессии следующие ограничения:

1) случайные величины $X$ имеют плотность распределения $g(x)$ по отношению к мере $\mu$, причем

$$
0<m \leqslant g(x) \leqslant M<\infty
$$

2) условная дисперсия

$$
r^{2}(x)=\mathbf{D}\{Y \mid X=x\} \leqslant \sigma^{2}<\infty
$$

3) поперечники Колмогорова $d_{N}(\mathbf{F}) \rightarrow 0, N \rightarrow \infty$.

Теорема 1.1. Пусть выполняются условия 1)-3). Тогда существуют оченки $\hat{f}_{n}(x)$ Функции регрессии $f(x)$ такие, что если число наблюдений $n \rightarrow \infty$, то

$$
\mathbf{P}\left\{\sup _{f \in \mathbf{F}} \mathbf{E}_{f}\left\{\left\|\hat{f}_{n}-f\right\|^{2} \mid X_{1}, X_{2}, \ldots, X_{n}\right\}=o(1)\right\} \longrightarrow 1
$$

Чтобы получить более точные границы скорости сходимости в (1.7), нам потребуются некоторые дополнительные индивидуальные характеристики приближающих $\mathbf{F}$ подпространств $M_{N}$. Во-первых, вместо поперечника Колмогорова мы будем часто рассматривать индивидуальную характеристику приближения $\mathbf{F}$ посредством $M_{N}$

$$
d\left(M_{N} ; \mathbf{F}\right)=\sup _{x \in \mathbf{F}} \inf _{y \in M_{N}}\|x-y\|
$$


Далее, положим

$$
A\left(M_{N} ; \mathbf{F}\right)=\inf \left\|\sum_{1}^{N} \varphi_{j}^{2}\right\|_{\infty},
$$

где нижняя грань берется по всем ортонормальным базисам $\left\{\varphi_{j}\right\}$ пространства $M_{N}$. Нам понадобятся еще Фурье уклонения в равномерной метрике множества $\mathbf{F}$ от $M_{N}$ при фиксированном ортонормальном базисе $\left\{\varphi_{j}\right\}$ пространства $M_{N}$ :

$$
\alpha\left(M_{N},\left\{\varphi_{j}\right\} ; \mathbf{F}\right)=\sup _{f \in \mathbf{F}}\left\|f-\sum_{1}^{N} \varphi_{j}\left(f, \varphi_{j}\right)\right\|_{\infty} .
$$

Ниже, когда ясно, о каких пространстве $M_{N}$ и базисе в нем идет речь, мы пишем для краткости вместо $d\left(M_{N} ; \mathbf{F}\right), A\left(M_{N} ; \mathbf{F}\right), \alpha\left(M_{N},\left\{\varphi_{j}\right\} ; \mathbf{F}\right)$ просто $d_{N}, A_{N}, \alpha_{N}$.

Теорема 1.2. Пусть подпространству $M_{N}$ отвечают введенные вьие характеристики $d_{N}, A_{N}$. Существуют оченки $f_{N}$ функции $f$ такuе, чmо

$$
\begin{aligned}
\mathbf{P} & \left\{\sup _{f \in \mathbf{F}} \mathbf{E}_{f}\left\{\left\|f-f_{N}\right\|^{2} \mid X_{1}, X_{2}, \ldots, X_{n}\right\} \geqslant c_{1} R\left(d_{N}^{2}+\sigma^{2} N n^{-1}\right)\right\} \\
& \leqslant c_{2}\left(R^{-1}+B_{N} n^{-1}\right) .
\end{aligned}
$$

Здесь $\sigma^{2}$ - граница условной дисперсии из (1.6), а постояннье $c_{i}$ зависят лишь от $M, m$ из (1.5).

Следствие 1.1. В условиях теоремы 1.2 существуют оценки $\hat{f}_{n}$ функции $f$ такие, что

$$
\begin{aligned}
\mathbf{P} & \left\{\sup _{f \in \mathbf{F}} \mathbf{E}_{f}\left\{\left\|\hat{f}_{n}-f\right\|^{2} \mid X_{1}, X_{2}, \ldots, X_{n}\right\} \geqslant c_{1} R\left(d_{N(n)}^{2}+\sigma^{2} N(n) n^{-1}\right)\right\} \\
& \leqslant c_{2}\left(R^{-1}+N(n) n^{-1}\right),
\end{aligned}
$$

где $N(n)$ минимизирует сумму $d_{N}^{2}+\sigma^{2} N n^{-1}$.

Использование характеристик $\alpha_{N}$ позволяет усилить неравенства теоремы 1.2 .

Теорема 1.3. Пусть подпространству $M_{N}$, как и выше, отвечают характеристики $d_{N}, A_{N}$, а также $\alpha_{N}$. Существуют оченки $f_{N}$ функиии $f$ такие, ито

$$
\begin{aligned}
\mathbf{P} & \left\{\sup _{f \in \mathbf{F}} \mathbf{E}_{f}\left\{\left\|f_{N}-f\right\|^{2} \mid X_{1}, X_{2}, \ldots, X_{n}\right\} \geqslant c_{1}\left(d_{N}^{2}+\sigma^{2} N n^{-1}\right)\right\} \\
& \leqslant c_{2}\left(\exp \left\{-c_{3}\left(n A_{N}^{-1}-N\right)\right\}+\exp \left\{-c_{4} n d_{N}^{2} \alpha_{N}^{-2}\right\}\right) .
\end{aligned}
$$

Константы с с зависят только от $M u$. 
Схема доказательства теорем 1.1-1.3 состоит в следующем. Пусть $M_{N}$ - какое-нибудь приближающее $\mathbf{F} N$-мерное подпространство, и пусть $\left\{\varphi_{j}\right\}$ - какой-нибудь ортонормальный базис в $M_{N}$. Дополняя его до полного базиса, разложим функцию $f$ в ряд Фурье, $f=\sum a_{j} \varphi_{j}$, $\sum_{N+1}^{\infty}\left|a_{j}\right|^{2} \leqslant d_{N}^{2}$. Рассматривая $a=\left(a_{1}, a_{2}, \ldots\right)$ как вектор в $l_{2}$, запишем вектор наблюдений $\mathbf{Y}_{n}$ в виде

$$
\mathbf{Y}_{n}=Z f+\xi
$$

где оператор $Z: l_{2} \rightarrow \mathbf{R}^{n}$ определяется матрицей $\left\|\varphi_{i}\left(X_{j}\right)\right\|, i=1,2, \ldots$, $j=1,2, \ldots, n$. Заметим, что компоненты вектора $\xi=\left(\xi_{1}, \xi_{2}, \ldots, \xi_{n}\right)$ не только независимы, но и условно независимы при условии, что фиксирован вектор $\left(X_{1}, X_{2}, \ldots, X_{n}\right)$.

Обозначим $E_{N}$ проектор в $l_{2}$ на $N$-мерное подпространство $\mathscr{E}_{N}$, натянутое на первые $N$ координат; через $E_{N}^{\perp}$ обозначим проектор на ортогональное дополнение к $\mathscr{E}_{N}$. Построим оценку наименьших квадратов для первых $N$ коэффициентов $a_{j}$ :

$$
\widehat{a}_{N}=\left(\widehat{a}_{1 N}, \widehat{a}_{2 N}, \ldots, \widehat{a}_{N N}\right)=\left(E_{N} Z^{*} Z E_{N}\right)^{-1} E_{N} Z^{*} \mathbf{Y}_{n}
$$

и оценим $f(x)$ посредством $f_{N}(x)=\sum_{1}^{N} a_{j N} \varphi_{j}(x)$. Так строятся оценки теорем 1.1-1.3. Для них

$$
\begin{aligned}
& \mathbf{E}\left\{\left\|f_{N}-f\right\| \mid X_{1}, X_{2}, \ldots, X_{n}\right\} \\
& \leqslant \sum_{N+1}^{\infty}\left|a_{j}\right|^{2}+\left\|\left(E_{N} Z^{*} Z E_{N}\right)^{-1}\right\|^{2}\left\|E_{N} Z^{*} Z E_{N}^{\perp} a\right\|^{2} \\
& \quad+\sigma^{2} \operatorname{tr}\left(E_{N} Z^{*} Z E_{N}\right)^{-1} .
\end{aligned}
$$

Здесь норма матрицы $A=\left\|a_{i j}\right\|, i, j=1,2, \ldots, N$, 一 это верхняя грань $|(A \xi, \xi)|$, взятая по всем векторам $\xi \in \mathbf{R}^{N}$ единичной длины, $\operatorname{atr} A$ означает след матрицы $A$. Анализ правой части неравенства (1.14) приводит к сформулированным выше результатам. Это доказательство содержится в разделе 2 . В разделе 3 эти теоремы применяются для исследования задачи, рассмотренной Ч. Стоуном в [15].

В работе [15] не было необходимости специально исследовать границы точности оценивания сверху, достаточно было использовать подобные границы, доказанные для случая $d=1$ (см. [7], [8], [14]). В более общей постановке такие границы определяются поперечниками Бернштейна и уже нуждаются в отдельном доказательстве. Соответствуюшие результаты излагаются в разделе 4. Наконец, в разделе 5 мы рассматриваем другой метод исследования возможных границ точности оценивания, основанный на поведении $\varepsilon$-энтропии множества $\mathbf{F}$.

Мой интерес к рассматриваемым здесь задачам возник под влиянием вопроса проф. W. Härdle о зависимости констант в работе [15] от 
размерности. Примеры пп. 3.1, 3.2 и пример 4.1 являются частичным ответом на этот вопрос. Пример п. 5.2 является ответом на вопрос, заданный мне проф. J. Horowitz'oм. Я весьма признателен W. Härdle и J. Horowitz'y за стимулирующее обсуждение данной работы.

2. Верхние границы. Доказательство теорем 1.1-1.3.

2.1. Как уже отмечалось, исходной точкой доказательств теорем 1.1-1.3 служит неравенство (1.14). Чтобы использовать это неравенство, мы должны сперва указать границы для норм матриц $E_{N} Z^{*} Z E_{N}$, $\left(E_{N} Z^{*} Z E_{N}\right)^{-1}$ и нормы вектора $E_{N} Z^{*} Z E_{N}^{\perp} a$.

Лемма 2.1. Пусть $M_{N}-$ какое-нибудь $N$-мерное подпростран-

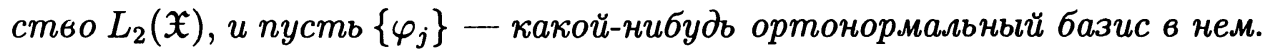
Обозначим $\lambda_{N}(X)=\lambda_{N}, \Lambda_{N}(X)=\Lambda_{N}$ минимальное и максимальное числа матрищы

$$
D_{N}=E_{N} Z^{*} Z E_{N}=\left\|\sum_{i=1}^{n} \varphi_{\nu}\left(X_{i}\right) \varphi_{\mu}\left(X_{i}\right)\right\|, \quad \nu, \mu=1, \ldots, N .
$$

Тогда

$$
\begin{aligned}
\mathbf{P}\left\{\Lambda_{N} \geqslant 4 M n\right\} & \leqslant c_{1} \exp \left\{-c_{2} n A_{N}^{-1}+c_{3} N\right\} \\
\mathbf{P}\left\{\lambda_{N} \leqslant 4^{-1} m n\right\} & \leqslant c_{4} \exp \left\{-c_{5} n A_{N}^{-1}+c_{6} N\right\}
\end{aligned}
$$

где $A_{N}=\sup _{x} \sum_{1}^{N} \phi_{j}^{2}(x)$, а постояннье с с зависят только от $m$ и $M$ из условия (1.5).

Д о к а з а т е л с т в о. Имеем, полагая $\xi=\left(\xi_{1}, \ldots, \xi_{n}\right)$,

$$
\begin{aligned}
& \lambda_{N}=\inf _{|\xi|=1} \sum_{i=1}^{n}\left|\sum_{\nu=1}^{N} \xi_{\nu} \varphi_{\nu}\left(X_{i}\right)\right|^{2}, \\
& \Lambda_{N}=\sup _{|\xi|=1} \sum_{i=1}^{n}\left|\sum_{\nu=1}^{N} \xi_{\nu} \varphi_{\nu}\left(X_{i}\right)\right|^{2} .
\end{aligned}
$$

При фиксированном $\xi$ случайные величины

$$
h_{i}^{2}(\xi)=\left|\sum_{\nu=1}^{N} \xi_{\nu} \varphi_{\nu}\left(X_{i}\right)\right|^{2}
$$

независимы и ограничены, $\left|h_{i}\right|^{2} \leqslant A_{N}$ (если $A_{N}=\infty$, то нечего и доказывать). В силу условия (1.5)

$$
m \leqslant \int_{\mathfrak{X}}\left|\sum_{\nu=1}^{N} \xi_{\nu} \varphi_{\nu}(x)\right|^{2} g(x) d \mu=\mathbf{E} h_{i}^{2}(\xi) \leqslant M .
$$


Поэтому для любого $t>0$

$$
\begin{aligned}
& \mathbf{P}\left\{\sum_{1}^{n} h_{i}^{2}(\xi)>2 M n\right\} \leqslant \mathbf{P}\left\{\sum_{1}^{n}\left(h_{i}^{2}-\mathbf{E} h_{i}^{2}\right) \geqslant M n\right\} \\
& \quad \leqslant \exp \{-t M n\}\left(\mathbf{E} \exp \left\{t\left(h_{i}^{2}-\mathbf{E} h_{i}^{2}\right)\right\}\right)^{n} \\
& \quad \leqslant \exp \{-t M n\}\left(1+\sum_{2}^{\infty} \frac{t^{\nu}}{\nu !} M A_{N}^{\nu-1}\right)^{n} \\
& \quad \leqslant \exp \{-t M n\}\left(1+2^{-1} M A_{n} t^{2} e^{t A_{N}}\right) \leqslant \exp \left\{-t M n+2^{-1} M A_{N} t^{2} e^{t A_{N}}\right\} .
\end{aligned}
$$

Полагая здесь $t=\left(2 A_{N}\right)^{-1}$, мы придем к неравенству

$$
\mathbf{P}\left\{\sum_{1}^{n} h_{i}^{2}(\xi)>2 M n\right\} \leqslant \exp \left\{-n\left(4 M A_{N}\right)^{-1}\right\} .
$$

Пусть $\xi=\left(\xi_{1}, \ldots, \xi_{N}\right)$ - какой-нибудь вектор из $\mathbf{R}^{N}$ с $|\xi|=1$. Обозначим $S_{\delta}(\xi)$ сферическую шапочку: $S_{\delta}(\xi)=\{\eta:|\xi+\eta|=1,|\eta| \leqslant \delta\}$. Положим $H(\zeta)=\sum_{1}^{n} h_{i}^{2}(\zeta)$. Тогда для всех $\eta=\left(\eta_{1}, \ldots, \eta_{N}\right) \in S_{\delta}(\xi)$

$$
\begin{aligned}
H(\xi+\eta) & \leqslant H(\xi)+\sum_{i=1}^{n}\left|\sum_{\nu=1}^{N} \eta_{\nu} \varphi_{\nu}\left(X_{i}\right)\right|\left|\sum_{\nu=1}^{N}\left(2 \xi_{\nu}+\eta_{\nu}\right) \varphi_{\nu}\left(X_{i}\right)\right| \\
& \leqslant H(\xi)+|\eta| \Lambda_{N}^{1 / 2}\left(4 H(\xi)+4 \sqrt{H(\xi) \Lambda_{N}}|\eta|+|\eta|^{2} \Lambda_{N}\right) \\
& \leqslant\left(\sqrt{H(\xi)}+\delta \sqrt{\Lambda_{N}}\right)^{2} .
\end{aligned}
$$

Выбирая $\delta=\frac{9}{10}-\frac{1}{\sqrt{2}}$, мы найдем из последнего неравенства, что либо $H(\xi)>2^{-1} \Lambda_{N}$, либо

$$
\sup _{\zeta \in S(\xi)} H(\zeta) \leqslant 9 \cdot 10^{-1} \Lambda_{N}<\Lambda_{N}
$$

Пусть $\xi^{1}, \ldots, \xi^{R}$ - максимальное множество точек единичной сферы $|\xi|=1$, отстоящих попарно друг от друга на расстояние, большее $\delta$. Тогда введенные выше сферические шапочки $S_{\delta}\left(\xi^{\nu}\right)$ покрывают всю единичную сферу и, значит, неравенство (2.6) не может выполняться для всех шапочек $S_{\delta}\left(\xi^{\nu}\right)$. Поэтому $\Lambda_{N} \leqslant 2 \max _{\nu} H\left(\xi^{\nu}\right)$. Далее, плошадь шапочки $S_{\delta}\left(\xi^{\nu}\right)$, равная

$$
\int_{x_{1}^{2}+\cdots+x_{N-1}^{2} \leqslant \delta^{2}} \frac{d x_{1} \cdots d x_{N-1}}{\sqrt{1-x_{1}^{2}-\cdots-x_{N-1}^{2}}},
$$

больше, чем $c e^{-N} \times\{$ плошадь единичной сферы $\}$, так что число шапочек покрытия $R \leqslant C e^{-N}$, где $c, C-$ положительные постоянные. Следовательно, в силу (2.5)

$$
\begin{aligned}
\mathbf{P}\left\{\Lambda_{N}>4 M n\right\} & \leqslant \sum_{\nu=1}^{R} \mathbf{P}\left\{H\left(\xi_{\nu}\right)>2 M n\right\} \\
& \leqslant c_{1} \exp \left\{-\left(4 A_{N}\right)^{-1} n+c_{2} N\right\} .
\end{aligned}
$$


Неравенство (2.1) доказано. Переходя к доказательству неравенства (2.2), отметим сперва, что, подобно (2.5),

$$
\begin{aligned}
\mathbf{P}\left\{\sum h_{i}^{2}(\xi)<2^{-1} m n\right\} & \leqslant \mathbf{P}\left\{\sum_{1}^{n}\left(h_{i}^{2}(\xi)-\mathbf{E} h_{i}^{2}(\xi)\right)<-2^{-1} m n\right\} \\
& \leqslant \exp \left\{-n m^{2}\left(2 M A_{N}\right)^{-1}\right\} .
\end{aligned}
$$

Рассмотрим опять сферическую шапочку $S_{\delta}(\xi)$. Как и выше, для $\eta \in S_{\delta}(\xi)$

$$
H(\xi+\eta) \geqslant H(\xi)-3 \delta \Lambda_{N},
$$

и потому, выбирая $\delta=m(48 M)^{-1}$, найдем, что

$$
\begin{aligned}
\mathbf{P}\left\{\lambda_{N} \leqslant 4^{-1} m n\right\} \leqslant & c_{1} \exp \left\{-n m^{2}\left(2 M A_{N}\right)^{-1}+c_{2} N\right\} \\
& +c_{3} \exp \left\{-\left(4 A_{N}\right)^{-1} n+N \ln 2\right\} .
\end{aligned}
$$

Лемма доказана.

2.2. Обратимся теперь к оценке нормы вектора $E_{N} Z^{*} Z E_{N}^{\perp} a$, где $a \in l_{2}$ - это вектор коэффициентов ряда Фурье $\sum_{\nu} a_{\nu} \varphi_{\nu}(x)$ функции $f$ по ортонормальному базису $\left\{\varphi_{\nu}\right\}$.

Лемма 2.2. Пусть $M_{N}$ - какое-нибудь $N$-мерное подпростран-

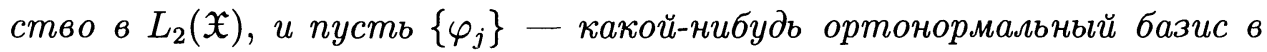
нем. Если $A_{N}=\left\|\sum \varphi_{j}^{2}\right\|_{\infty}$, то для всех $a \in \mathbf{F}$

$$
\mathbf{P}\left\{\left\|E_{N} Z^{*} Z E_{N}^{\perp} a\right\|^{2}>R n d_{N}^{2}\right\} \leqslant R^{-1}+c_{1} \exp \left\{-c_{2} n A_{N}^{-1}+c_{3} N\right\} .
$$

Д ок а з а т ел ь с т в о. Имеем

$$
\begin{aligned}
\left\|E_{N} Z^{*} Z E_{N}^{\perp} a\right\|^{2} & =\sup _{\{\xi:|\xi|=1\}}\left(E_{N} Z^{*} Z E_{N}^{\perp} a, \xi\right)^{2} \\
& =\sup _{\xi}\left|\sum_{i=1}^{n} \sum_{\nu=1}^{N} \xi_{\nu} \varphi_{\nu}\left(X_{i}\right) \sum_{\nu=N+1}^{\infty} a_{\nu} \varphi_{\nu}\left(X_{i}\right)\right|^{2} \\
& \leqslant \Lambda_{N} \sum_{i=1}^{n}\left|\sum_{\nu=N+1}^{\infty} a_{\nu} \varphi_{\nu}\left(X_{i}\right)\right|^{2}
\end{aligned}
$$

В силу условия (1.5)

$$
\mathbf{E}\left|\sum_{\nu=N+1}^{\infty} a_{\nu} \varphi_{\nu}^{2}\left(X_{i}\right)\right|^{2}=\int_{\mathfrak{X}}\left|\sum_{N+1}^{\infty} a_{\nu} \varphi_{\nu}(x) g(x)\right|^{2} d \mu \leqslant m \sum_{N+1}^{\infty}\left|a_{\nu}\right|^{2} \leqslant m d_{N}^{2} .
$$

Отсюда, из неравенства Чебышева и неравенства (2.1) уже следует неравенство (2.9). Лемма доказана.

Лемма 2.3. Пусть $M_{N}$ - какое-нибудь $N$-мерное подпростран-

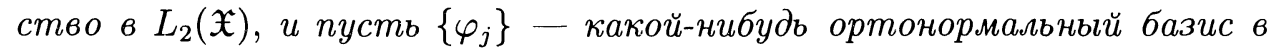
нем. Пусть $A_{N}, \alpha_{N}$ - характеристики (1.8), (1.9), соответствующие 
$\left(M_{N},\left\{\varphi_{j}\right\}\right)$. Тогда для любого $f \in \mathbf{F}, f(x)=\sum_{\nu} a_{\nu} \varphi_{\nu}(x)$, справедливо неравенство

$$
\begin{aligned}
& \mathbf{P}\left\{\left\|E_{N} Z^{*} Z E_{N}^{\perp} a\right\|^{2}>2 M n d_{N}^{2}\right\} \\
& \quad \leqslant c_{1} \exp \left\{-c_{2} n A_{N}^{-1}+c_{3} N\right\}+\exp \left\{-c_{4} n d_{N}^{2} \alpha_{N}^{-2}\right\} .
\end{aligned}
$$

Д ок а з а т е л с т в о. Обратимся снова к неравенству (2.10). Случайные величины $\eta_{i}=\left|\sum_{\nu=N+1}^{\infty} a_{\nu} \varphi_{\nu}\left(X_{i}\right)\right|^{2}, i=1, \ldots, n$, независимы, ограничены сверху величиной $\alpha_{N}^{2}$ и $\mathbf{E} \eta_{i} \leqslant M d_{N}^{2}$. Поэтому

$$
\begin{aligned}
\mathbf{P}\left\{\sum_{1}^{n} \eta_{i}>2 M n d_{N}^{2}\right\} & \leqslant e^{-2 M t n d_{N}^{2}}\left(\mathbf{E} e^{\eta_{i}-\mathbf{E} \eta_{i}}\right)^{n} \\
& \leqslant e^{-2 M n t d_{N}^{2}}\left(1+\sum_{k=2}^{\infty} \frac{\mathbf{E}\left(\eta_{i}-\mathbf{E} \eta_{i}\right)^{k} t^{k}}{k !}\right)^{n} \\
& \leqslant e^{-2 M t n d_{N}^{2}}\left(1+2 t^{2} M d_{N}^{2} \alpha_{N}^{2} e^{2 t \alpha_{N}^{2}}\right)^{n} \\
& \leqslant \exp \left\{-2 M n d_{N}^{2} t+2 M n t^{2} d_{N}^{2} \alpha_{N}^{2} e^{2 t \alpha_{N}^{2}}\right\} .
\end{aligned}
$$

Полагая здесь $t=\lambda \alpha_{N}^{-2}$ и выбирая $\lambda$ так, чтобы максимизировать $\lambda-e^{2 \lambda}$, придем к неравенству (2.11). Лемма доказана.

2.3. Перейдем к доказательству теорем 1.1-1.3. Докажем сперва теорему 1.2. Выбрав какое-нибудь $N$-мерное подпространство $M_{N}$ и базис $\left\{\varphi_{j}\right\}$ в нем, рассмотрим оценку $f_{N}$, определенную посредством (1.13), и напишем для нее неравенство (1.14). В силу леммы 2.1

$$
\mathbf{P}\left\{\left\|\left(E_{N} Z^{*} Z E_{N}\right)^{-1}\right\|>4 m^{-1} n^{-1}\right\} \leqslant c_{1} \exp \left\{c_{2} n A_{N}^{-1}+c_{3} N\right\} .
$$

Отсюда и из леммы 2.2 вытекает, что для любого $R>0$

$$
\begin{aligned}
& \mathbf{P}\left\{\left\|\left(E_{N} Z^{*} Z E_{N}\right)^{-1}\right\|^{2}\left\|E_{N} Z^{*} Z E_{N}^{\perp} a\right\|^{2}>R d_{N}^{2}\right\} \\
& \quad \leqslant c_{0} R^{-1}+c_{1} \exp \left\{-c_{2} n A_{N}^{-1}+c_{3} N\right\} .
\end{aligned}
$$

По лемме 2.1

$$
\begin{aligned}
& \mathbf{P}\left\{\operatorname{tr}\left(E_{N} Z^{*} Z E_{N}\right)^{-1}>4 N m^{-1} n^{-1}\right\} \leqslant \mathbf{P}\left\{\lambda_{N}<4^{-1} m n\right\} \\
& \quad \leqslant c_{1} \exp \left\{-c_{2} n A_{N}^{-1}+c_{3} N\right\} .
\end{aligned}
$$

Применяя неравенства $(2.13),(2.14)$ к (1.15), найдем, что

$$
\begin{aligned}
& \mathbf{P}\left\{\mathbf{E}\left\{\left\|f-f_{N}\right\|^{2} \mid X_{1}, \ldots, X_{n}\right\} \geqslant R d_{N}^{2}+4 \sigma^{2} N m^{-1} n^{-1}\right\} \\
& \quad \leqslant R^{-1}+c_{1} \exp \left\{-c_{2} n A_{N}^{-1}+c_{3} N\right\} .
\end{aligned}
$$

Теорема 1.2 доказана. Теорема 1.3 доказывается точно так же, только вместо неравенства леммы 2.2 нужно использовать неравенство леммы 2.3 . 
Докажем теорему 1.1. В условиях теоремы 1.1 можно выбрать такие $N$-мерное подпространство $M_{N}$ и базис $\left\{\varphi_{j}\right\}$ в нем, что расстояние $\mathbf{F}$ от $M_{N}$ не превзойдет $2 d_{N}, d_{N}$ - поперечник Колмогорова, а $\left\|\sum_{1}^{N} \varphi_{j}\right\|_{\infty}=$ $A_{N}<\infty$. Полагая в неравенстве теоремы $1.2 R=d_{N}^{-1}$, мы найдем, что

$$
\mathbf{P}\left\{\mathbf{E}\left\{\left\|f-f_{N}\right\|^{2} \mid X_{1}, \ldots, X_{n}\right\} \geqslant d_{N}\right\} \leqslant c_{0} d_{N}+c_{1} \exp \left\{-c_{2} n A_{N}^{-1}+c_{3} N\right\} .
$$

Выбирая теперь $N=N(n) \rightarrow \infty$, когда $n \rightarrow \infty$, но настолько медленно, чтобы правая часть (2.16) стремилась к нулю, мы придем к утверждению теоремы.

\section{3. Примеры.}

3.1. Здесь мы применим результаты предыдущего раздела к задаче, рассмотренной Стоуном в [15]. Мы начнем с наиболее простого случая периодических функций. Допустим, что $\mathfrak{X}$ - это единичный куб $[0,1]^{d}$ евклидова пространства $\mathbf{R}^{d}$. Предположим, далее, что $\mathbf{F}-$ это класс функций $f(x), x=\left(x_{1}, \ldots, x_{d}\right)$, представимых в виде

$$
f(x)=f_{1}\left(x_{1}\right)+\cdots+f_{d}\left(x_{d}\right),
$$

где все определенные в $\mathbf{R}^{1}$ функции $f_{j}(x)$ 1-периодичны, принадлежат $L_{2}[0,1]$, имеют там $r \geqslant 0$ производных, а последняя производная $f_{j}^{(r)}$ удовлетворяет в $L_{2}[0,1]$ условию Гёльдера порядка $\alpha, 0<\alpha \leqslant 1$, т.е.

$$
\int_{0}^{1}\left|f^{(r)}(x+h)-f^{(r)}(x)\right|^{2} d x \leqslant L|h|^{2 \alpha} .
$$

Таким образом, класс $\mathbf{F}=\mathbf{F}(\beta, L)$ определяется двумя параметрами $\beta=r+\alpha$ и $L$.

Теорема 3.1. При соблюдении перечисленных выще условий существуют такие оченки $\hat{f}_{n}$ бункиии регрессии $f$, что

$$
\begin{aligned}
\mathbf{P} & \left\{\sup _{f \in \mathbf{F}} \mathbf{E}_{f}\left\{\left\|f-\hat{f}_{n}\right\|^{2} \mid X_{1} \ldots, X_{n}\right\}>c_{0} d n^{-2 \beta /(2 \beta+1)}\right\} \\
& \leqslant c_{1} \exp \left\{-c_{2}\left(d^{-1} n^{2 \beta /(2 \beta+1)}-d n^{1 /(2 \beta+1)}\right)\right\} .
\end{aligned}
$$

При этом постоянные $c_{i}$ могут зависеть от $m, M$ и $\sigma$ из (1.5) $u$ (1.6), a также от параметров $\beta, L$, но не от размерности $d$.

Д о к а з а т е л ь с т в о. Мы выведем (3.2) из теоремы 1.3. Разложим функции $f_{j}(x)$ в ряд Фурье, $f_{j}(x)=b_{0 j}+\sum_{l=1}^{\infty} \sqrt{2}\left(a_{l j} \sin (2 \pi l x)+\right.$ $\left.b_{l j} \cos (2 \pi l x)\right)$. В пространстве $L_{2}[0,1]$ тригонометрическими полиномами наилучшего приближения служат отрезки ряда Фурье. Поэтому из условия (3.1) следует (см., например, $[1$, с. 252]), что

$$
\int_{0}^{1}\left|f_{j}(x)-b_{0 j}-\sum_{l=1}^{k} \sqrt{2}\left(a_{l j} \sin (2 \pi l x)+b_{l j} \cos (2 \pi l x)\right)\right|^{2} d x \leqslant 9 L k^{-2 \beta} .
$$


Рассмотрим подпространство $M_{N}$ размерности $N=1+2 d k$ пространства $L_{2}(\mathfrak{X})$, натянутое на функции $\left\{1, \sin \left(2 \pi l x_{j}\right), \cos \left(2 \pi l x_{j}\right)\right.$, $j=1, \ldots, d, l=1, \ldots, k\}$. В силу $(3.3)$

$$
d_{N}^{2}\left(M_{N}\right) \leqslant 9 L d k^{-\beta} \leqslant 9 \cdot 2^{2 \beta} L d^{1+2 \beta}(N-1)^{-2 \beta} .
$$

Очевидно,

$$
A_{N}\left(M_{N}\right) \leqslant 1+2 k d=N
$$

В силу (3.3)

$$
\begin{aligned}
\alpha_{N}\left(M_{N},\left\{\varphi_{j}\right\}\right) & \leqslant \sum_{j=1}^{d} \sum_{l=k+1}^{\infty} \sqrt{2}\left(\left|a_{l j}\right|+\left|b_{l j}\right|\right) \\
& \leqslant C \sum_{j=1}^{d} \sum_{r=\log _{2} k}^{\infty} 2^{r / 2} \sum_{l=2^{r}}^{2^{r+1}}\left(\left|a_{l j}\right|^{2}+\left|b_{l j}\right|^{2}\right)^{1 / 2} \\
& \leqslant C k^{-(\beta-1 / 2)} \leqslant C d^{\beta+1 / 2} N^{-(\beta-1 / 2)}
\end{aligned}
$$

Из теоремы 1.3 следует тогда, что существуют оценки $\hat{f}_{N}$ функции $f$ такие, что

$$
\begin{aligned}
& \mathbf{P}\left\{\sup _{f} \mathbf{E}_{f}\left\{\left\|f-\hat{f}_{N}\right\|^{2} \mid X_{1}, \ldots, X_{n}\right\}>c_{0}\left(d^{1+2 \beta} N^{-2 \beta}+\sigma^{2} N n^{-1}\right)\right\} \\
& \quad \leqslant c_{1} \exp \left\{-c_{2}\left(n N^{-1}-N\right)\right\}+c_{3} \exp \left\{-c_{4} n N^{-1}\right\} .
\end{aligned}
$$

Полагая здесь $N=N(n) \sim d n^{1 /(2 \beta+1)}$ и $\hat{f}_{n}=\hat{f}_{N(n)}$, придем к неравенству теоремы.

3.2. В этом пункте мы рассмотрим ту же задачу, что и в п. 3.1 , но откажемся от предположения о периодичности $f$. Таким образом, теперь $\mathbf{F}$ состоит из всех функций $f \in L_{2}[0,1]$, удовлетворяющих (3.1) с $\beta>\frac{1}{2}$. При этом, как обычно (см., например, [13, с. 175]), предполагается, что интегрирование в (3.1) ведется лишь по таким $x$, для которых и $x+h \in[0,1]$.

Теорема 3.2. Пусть выполняются все перечисленнье в этом пункте условия и основные условия раздела 1. Тогда найдутся такие оченки $\hat{f}_{n}$ Функиии регрессии $f$, что

$$
\begin{aligned}
& \mathbf{P}\left\{\sup _{f \in \mathbf{F}} \mathbf{E}_{f}\left\{\left\|f-\hat{f}_{n}\right\|^{2} \mid X_{1}, \ldots, X_{n}\right\}>c_{0} d n^{-2 \beta /(2 \beta+1)}\right\} \\
& \quad \leqslant c_{1} \exp \left\{-c_{2}\left(d^{-1} n^{2 \beta /(2 \beta+1)}-d n^{1 /(2 \beta+1)}\right)\right\} .
\end{aligned}
$$

При этом постоянные $c_{i}$ могут зависеть от $m, M u \sigma$ из (1.5) $u$ (1.6), а также от параметров $\beta, L$, но не от размерности $d$.

Д о к а з а т ел ь с т в о. Если $\beta \leqslant 1$, то функции $f_{j}$ можно продолжить периодически на всю вещественную прямую с сохранением гладкости $\beta$. Поэтому для $\beta \leqslant 1$ теорема 3.2 следует из теоремы 3.1 . В обшем 
случае для любой функции $f_{j}$ из (3.1) можно подобрать полином $P_{j}(x)$ степени не выше $2 \beta$ такой, что функция $g_{j}(x)=f_{j}(x)-P_{j}(x)$ допускает периодическое продолжение гладкости $\beta$.

Рассматривая ряд Фурье $\sum_{l=0}^{\infty} \sqrt{2}\left(a_{l k} \sin (2 \pi l x)+b_{l k} \cos (2 \pi l x)\right)$ функции $x^{k}$, найдем, что

$$
a_{l k}=\sqrt{2} \int_{0}^{1} x^{k} \sin (2 \pi l x) d x=\frac{1}{\pi \sqrt{2} l}+O\left(l^{-2}\right) .
$$

Аналогично, $b_{l k}=O\left(l^{-2}\right)$. Поэтому проекция $\theta_{k}(x)$ функции $x^{k}$ в $L_{2}[0,1]$ на подпространство, порожденное функциями $\{\cos (2 \pi l x), \sin (2 \pi l x)$, $l \geqslant N+1\}$, обладает следующими свойствами. Во-первых,

$$
\left\|\theta_{k}\right\|^{2}=\frac{1}{2 \pi^{2}} \sum_{N+1}^{\infty} \frac{1}{l^{2}}+O\left(N^{-3}\right)=\frac{1}{2 \pi^{2} N}(1+O(1)) .
$$

Во-вторых, в связи с тем, что суммы рядов $\sum_{l=r}^{\infty} \sin (2 \pi l x) / l$ равномерно ограничены по $r$ и $x$ (см., например, [4, с. 293]), все функции $\theta_{k}, k \leqslant 2 \beta$, равномерно ограничены, $\left\|\theta_{k}\right\| \leqslant c_{k}$. Поэтому если ортогонализировать и отнормировать систему функций $\theta_{1}, \theta_{2}, \ldots$, то соответствующие ортонормальные функции $\hat{\varphi}_{j}, j \leqslant 2 \beta$, будут ограничены сверху величиной $c \sqrt{N}$, так что и

$$
\sum_{j} \widehat{\varphi}_{j}^{2}(x) \leqslant c N
$$

Рассмотрим теперь подпространство $M_{N}$, натянутое на функции $\left\{x_{j}^{k}, 0 \leqslant k \leqslant 2 \beta, \sin \left(2 \pi l x_{j}\right), \cos \left(2 \pi l x_{j}\right), j=1, \ldots, d, l \leqslant N /(2 d)\right\}$. Система функций $\widehat{\varphi}_{k}\left(x_{j}\right), \sqrt{2} \sin \left(2 \pi l x_{j}\right), \sqrt{2} \cos \left(2 \pi l x_{j}\right)$ образует ортонормальный базис в $M_{N}$, для которого выполняются неравенства типа (3.4)-(3.6). Из этих неравенств теорема 3.2 выводится точно так же, как теорема 3.1 из неравенств (3.4)-(3.6).

4. Границы снизу. При выводе нижних границ точности оценивания мы ограничимся случаем, когда при фиксированных $X_{1}, \ldots, X_{n}$ разности

$$
\xi_{i}=Y_{i}-\mathbf{E}\left\{Y_{i} \mid X_{i}\right\}=Y_{i}-f\left(X_{i}\right)
$$

имеют нормальное распределение со средним 0 и дисперсией $D^{2}\left(X_{i}\right)$. Будем предполагать в дальнейшем, что дополнительно к (1.6) также и

$$
\inf _{x} r^{2}(x)=\sigma_{0}>0 .
$$

Теорема 4.1. Пусть подпространству $M_{N+1}$ соответствуют характеристики $b_{N}$ и $A_{N+1}$. Eсли $b_{N}\left(n N^{-1}\right)^{1 / 2} \geqslant 1$, тогда

$$
\begin{aligned}
& \mathbf{P}\left\{\inf _{\hat{f} \sup _{f \in \mathbf{F}}} \mathbf{E}_{f}\left\{\|\hat{f}-f\| \mid X_{1}, \ldots, X_{n}\right\} \geqslant c_{1} \sup _{N}\left(N n^{-1}\right)\right\} \\
& \quad \geqslant 1-\exp \left\{-c_{2}\left(n A_{N+1}^{-1}-N\right)\right\},
\end{aligned}
$$


где положительные постояннье $c_{i}>0$ зависят только от $m, M u \sigma$ из условий (1.5), (1.6).

Д ок а з а тел в с т в о. Пусть $M_{N}$ - какое-либо подпространство, содержашее $N$-мерный диск $B_{N} \subseteq \mathbf{F}$ радиуса $b_{N}$ (см. (1.4)). Без потери общности можно считать, что центр диска расположен в нуле. Очевидно, что для любой оценки $\hat{f}$ функции $f$ и всех $t>0$

$$
\begin{aligned}
& \mathbf{P}\left\{\sup _{f \in \mathbf{F}} \mathbf{E}_{f}\left\{\|f-\hat{f}\|^{2} \mid X_{1}, \ldots, X_{n}\right\}>t\right\} \\
& \quad \leqslant \mathbf{P}\left\{\sup _{f \in B_{N}} \mathbf{E}_{f}\left\{\|f-\hat{f}\|^{2} \mid X_{1}, \ldots, X_{n}\right\}>t\right\} .
\end{aligned}
$$

Все функции $f \in B_{N}$ можно записать в виде $f(x)=\sum_{1}^{N} a_{j} \varphi_{j}(x)$, где $\varphi_{j}$ какой-нибудь ортонормальный базис в $M_{N}$. Если ограничиться только функциями $f \in B_{N}$, то наблюдения $\mathbf{Y}$ можно записать в виде

$$
\mathbf{Y}=Z_{N} a+\xi, \quad Z_{N}=\left\|\varphi_{j}\left(X_{i}\right)\right\|, \quad a=\left(a_{1}, \ldots, a_{N}\right)
$$

$\xi=\left(\xi_{1}, \ldots, \xi_{n}\right)$ - условно нормальный случайный вектор со средним 0 и диагональной корреляционной матрицей

$$
R=\operatorname{diag}\left\{r^{2}\left(X_{1}\right), \ldots, r^{2}\left(X_{n}\right)\right\} .
$$

Лемма 4.1. Пусть наблюдение $Y$ - это $N$-мерный гауссовский случайный вектор, представимый в виде $Y=a+\xi$, где неизвестный параметр $a \in \mathbf{R}^{N}$, а шум $\xi$ есть $N$-мерный гауссовский вектор со средним нуль и невырожденной коррелячионной матрицей $R$. Обозначим $\lambda_{1}, \ldots, \lambda_{N}$ собственные числа матрицы $R$. Тогда для любой оченки $\widehat{a}$ вектора а

$$
\sup _{|a| \leqslant r} \mathbf{E}_{a}|a-\widehat{a}|^{2} \geqslant \frac{1}{2} \frac{1}{\sqrt{2 \pi}} \sum_{j=1}^{N} \lambda_{j} \int_{-r / 2 \sqrt{N \lambda_{j}}}^{r / 2 \sqrt{N \lambda_{j}}} a^{2} e^{-a^{2} / 2} d a .
$$

В частности, если для всех $j$ вьполняются неравенстьва $r \geqslant \sqrt{N \lambda_{j}}$, то

$$
\sup _{|a| \leqslant r} \mathbf{E}_{a}|a-\widehat{a}|^{2} \geqslant \frac{1}{2 \sqrt{2 \pi}} \int_{-1 / 2}^{1 / 2} a^{2} e^{-a^{2} / 2} d a \operatorname{tr} R .
$$

Д ок а зате л ь с т в о. Обозначим $U$ ортогональную матрицу, приводящую $R$ к диагональному виду, так что $U^{*} R U=\Lambda=$ $\operatorname{diag}\left\{\lambda_{1}, \ldots, \lambda_{N}\right\}$. Будем считать для определенности, что $\lambda_{1} \leqslant \cdots \leqslant \lambda_{N}$. Обозначая $S$ шар $\{a:|a| \leqslant r\}$, найдем, что

$$
\begin{aligned}
I=\sup _{a \in S} \mathbf{E}_{a}|a-\widehat{a}|^{2} & =\sup _{a \in S}(2 \pi)^{-N / 2}(\operatorname{det} R)^{-1 / 2} \\
\times & \int_{\mathbf{R}^{N}}|a-\widehat{a}(y)|^{2} \exp \left\{-\frac{1}{2}\left(R^{-1}(y-a),(y-a)\right)\right\} d y
\end{aligned}
$$




$$
\begin{aligned}
= & \sup _{a \in S}(2 \pi)^{-n / 2}(\operatorname{det} R)^{-1 / 2} \\
& \times \int_{\mathbf{R}^{N}}|U a-\widehat{a}(U y)|^{2} \exp \left\{-\frac{1}{2}\left(R^{-1}(U y-U A),(U a-U y)\right)\right\} d y \\
= & \sup _{a \in S}(2 \pi)^{-n / 2}(\operatorname{det} \Lambda)^{-1 / 2} \\
& \times \int_{\mathbf{R}^{N}}\left|a-U^{*} \widehat{a}(U y)\right|^{2} \exp \left\{-\frac{1}{2}\left(\Lambda^{-1}(a-y),(a-y)\right)\right\} d y .
\end{aligned}
$$

Обозначим $\Gamma N$-мерный куб, вписанный в $S$. Очевидно,

$$
\begin{aligned}
I \geqslant & \frac{1}{\operatorname{mes} \Gamma} \int_{\Gamma} d a(2 \pi)^{-N / 2}(\operatorname{det} \Lambda)^{-1 / 2} \\
& \times \int_{\mathbf{R}^{N}}\left|a-\widehat{a}_{1}(y)\right|^{2} \exp \left\{-\frac{1}{2}\left(\Lambda^{-1}(y-a),(y-a)\right)\right\} d y .
\end{aligned}
$$

(Заметим во избежание недоразумения, что функция $\widehat{a}_{1}(\mathbf{Y})=U^{*} \widehat{a}(U \mathbf{Y})$, вообще говоря, не является статистикой, поскольку $R$, а значит, и $U$ не предполагаются известными, но это обстоятельство нисколько не влияет на выводы, следуюшие из (4.7).)

Обозначим $\Gamma_{j}(N-1)$-мерный куб $\left\{\left|a_{1}\right| \leqslant r / \sqrt{N}, \ldots,\left|a_{j-1}\right| \leqslant\right.$ $\left.r / \sqrt{N},\left|a_{j+1}\right| \leqslant r / \sqrt{N}, \ldots,\left|a_{N}\right| \leqslant r / \sqrt{N}\right\}$, через $\Lambda_{j}$ обозначим матрицу $\Lambda$ с удаленными $j$-й строкой и $j$-м столбцом. Положим $d \mathbf{y}_{j}=$ $d y_{1} \cdots d y_{j-1} d y_{j+1} \cdots d y_{N}, d \mathbf{a}_{j}=d a_{1} \cdots d a_{j-1} d a_{j+1} \cdots d a_{N}$. Из (4.8) следует тогда, что

$$
\begin{aligned}
& I \geqslant \sum_{j=1}^{N} \frac{1}{\operatorname{mes} \Gamma_{j}} \frac{1}{(2 \pi)^{(N-1) / 2} \sqrt{\operatorname{det} \Lambda_{j}}} \\
& \times \int_{\mathbf{R}^{N-1}} \int_{\Gamma_{j}} \exp \left\{-\frac{1}{2} \sum_{k \neq j} \lambda_{k}^{-1}\left|y_{k}-a_{k}\right|^{2}\right\} d \mathbf{y}_{j} d \mathbf{a}_{j} \\
& \times \frac{\sqrt{N}}{2 r} \int_{-r / \sqrt{N}}^{r / \sqrt{N}} \frac{d a_{j}}{\sqrt{2 \pi \lambda_{j}}} \int_{-\infty}^{\infty}\left|a_{j}-\widehat{a}_{1 j}\right|^{2} e^{\left(y_{j}-a_{j}\right)^{2} /\left(2 \lambda_{j}\right)} d y_{j} \\
&= \sum_{j=1}^{N} \frac{1}{\operatorname{mes} \Gamma_{j}} \frac{1}{(2 \pi)^{(N-1) / 2} \sqrt{\operatorname{det} \Lambda_{j}}} \\
& \times \int_{\mathbf{R}^{N-1}} \int_{\Gamma_{j}} \exp \left\{-\frac{1}{2} \sum_{k \neq j} \lambda_{k}^{-1}\left|y_{k}-a_{k}\right|^{2}\right\} d \mathbf{y}_{j} d \mathbf{a}_{j} \\
& \times\left\{\lambda_{j} \frac{\sqrt{\lambda_{j} N}}{2 r \sqrt{2 \pi}} \int_{-\infty}^{\infty} d y_{j} \int_{r / \sqrt{N \lambda_{j}}}^{r / \sqrt{N \lambda_{j}}}\left|a_{j}-\lambda_{j}^{-1 / 2}\left(y_{j} \sqrt{\lambda_{j}}\right)\right|^{2}\right. \\
&\left.\times e^{-\left(y_{j}-a_{j}\right)^{2} / 2} d a_{j}\right\} .
\end{aligned}
$$

Заметим, что при всех $B, b>0$

$$
\int_{-B}^{B}|y-b|^{2} e^{-y^{2} / 2} d y \geqslant \int_{-B}^{B} y^{2} e^{-y^{2} / 2} d y .
$$


Поэтому заключенный в фигурные скобки двойной интеграл $j$-го слагаемого в правой части (4.9) не меньше, чем

$$
\begin{aligned}
& \frac{\lambda_{j}}{\sqrt{2 \pi}} \int_{-r / 2 \sqrt{N \lambda_{j}}}^{r / 2 \sqrt{N \lambda_{j}}} \frac{\sqrt{N \lambda_{j}}}{2 r} d y \int_{-r / 2 \sqrt{N \lambda_{j}}}^{r / 2 \sqrt{N \lambda_{j}}}\left|a_{j}-\lambda_{j}^{-1 / 2} \widehat{a}_{1 j}+y_{j}\right|^{2} e^{-a_{j}^{2} / 2} d a_{j} \\
& \geqslant \frac{\lambda_{j}}{2 \sqrt{2 \pi}} \int_{-r / 2 \sqrt{N \lambda_{j}}}^{r / 2 \sqrt{N \lambda_{j}}} a_{j}^{2} e^{a_{j}^{2} / 2} d a_{j} .
\end{aligned}
$$

Следовательно,

$$
\sup _{|a| \leqslant r} \mathbf{E}_{a}|a-\widehat{a}|^{2} \geqslant \frac{1}{2 \sqrt{2 \pi}} \sum_{j=1}^{N} \lambda_{j} \int_{-r / 2 \sqrt{N \lambda_{j}}}^{r / 2 \sqrt{N \lambda_{j}}} a^{2} e^{-a^{2} / 2} d a .
$$

Лемма доказана.

Лемма 4.2. Пусть $N$-мерный параметр а оченивается по наблюдению

$$
Y=Z a+\xi
$$

где $\xi-n$-мерный гауссовский вектор со средним нуль и невырожденной корреляционной матрицей $R, n \geqslant N$. Матрица $Z$ такова, что матрича $Z^{*} Z$ строго положительна. Тогда, какова бы ни была оченка $\widehat{a}$ вектора $a$,

$$
\sup _{|a| \leqslant r} \mathbf{E}_{a}|a-\widehat{a}|^{2} \geqslant \frac{1}{2 \sqrt{2 \pi}} \sum_{j=1}^{N} \lambda_{j} \int_{-r / 2 \sqrt{N \lambda_{j}}}^{r / 2 \sqrt{N \lambda_{j}}} a^{2} e^{-a^{2} / 2} d a,
$$

где $\lambda_{1}, \ldots, \lambda_{N}$ суть собственные числа матриць $\left(Z^{*} R^{-1} Z\right)^{-1}$. В частности, если для всех $j$ отночения $r / \sqrt{N \lambda_{j}} \geqslant 1$, то

$$
\sup _{|a| \leqslant r} \mathbf{E}_{a}|a-\widehat{a}|^{2} \geqslant \frac{1}{2 \sqrt{2 \pi}} \int_{-1 / 2}^{1 / 2} a^{2} e^{-a^{2} / 2} d a \cdot \operatorname{tr}\left(Z^{*} R^{-1} Z\right)^{-1}
$$

Д ока з а тель с т в о. Положим $K=R^{-1 / 2} Z$. Тогда

$$
Y^{\prime}=\left(K^{*} K\right)^{-1} K^{*} R^{-1 / 2} Y=a+\xi^{\prime}
$$

где $\xi^{\prime}-N$-мерный гауссовский вектор с корреляционной матрицей $R^{\prime}=\left(K^{*} K\right)^{-1}=\left(Z^{*} R^{-1} Z\right)^{-1}$. Если $R$ есть известная матрица, то, как легко видеть, $Y^{\prime}$ есть достаточная статистика. В силу теоремы Блэкуэлла-Колмогорова-Рао можно считать, что $\widehat{a}$ есть функция $Y^{\prime}$, и (4.13) следует из леммы 4.1. Если матрица $R$ не известна, все равно соответствующие неравенства, приводящие к (4.13), остаются в силе (ср. выше (4.7), (4.8)) (интуитивно, конечно, ясно, что точность оценивания при неизвестной матрице $R$ не может быть выше, чем при известной). 
Лемма 4.3. Обозначим $\mu_{1}, \ldots, \mu_{N}$ собственнье числа матрицы $\left(Z_{N}^{*} D^{-1} Z_{N}\right)^{-1}$. Если для всех $j=1, \ldots, N$ отношения $b_{N} / \sqrt{N \mu_{j}} \geqslant 1$, то, какова бы ни была оченка $\hat{f}$ функции регрессии $f$,

$$
\begin{aligned}
& \sup _{f \in B_{N}} \mathbf{E}_{f}\left\{\|\hat{f}-f\|^{2} \mid X_{1}, \ldots, X_{n}\right\} \\
& \geqslant \frac{1}{2 \sqrt{2 \pi}} \int_{-1 / 2}^{1 / 2} u^{2} e^{-u^{2} / 2} d u \operatorname{tr}\left(Z_{N}^{*} D^{-1} Z_{N}\right)^{-1} .
\end{aligned}
$$

Д о к а з а т е л ь с т в о. Лемма 4.3 следует из (4.2) и леммы 4.2 .

Обращаясь к доказательству теоремы, заметим, что $\mu_{1} \geqslant \sigma_{0}^{2} / \Lambda_{N}$, $\mu_{N} \leqslant \sigma^{2} / \lambda_{N}$, где, как и в разделе $2, \Lambda_{N}, \lambda_{N}$ означают максимальное и минимальное собственные числа матрицы $Z_{N}^{*} Z_{N}$. В силу леммы 2.1 с вероятностью, большей, чем $1-c_{1} \exp \left\{-c_{2} n A_{N}^{-1}+c_{3} N\right\}$, будут выполняться неравенства $\mu_{1} \geqslant c n^{-1}, \mu_{N} \leqslant C n^{-1}$, где $c, C$ - положительные постоянные, зависящие лишь от $m, M, \sigma, \sigma_{0}$. Поэтому найдутся такие постоянные $c^{\prime}>0, C^{\prime}>0$, что с вероятностью, большей $1-c_{1} \exp \left\{-c_{2} n A_{N}^{-1}+c_{3} N\right\}$, будут выполняться неравенства

$$
c^{\prime} b_{N} \sqrt{\frac{n}{N}} \leqslant \frac{b_{N}}{\sqrt{N \lambda_{j}}} \leqslant C^{\prime} b_{N} \sqrt{\frac{n}{N}}, \quad j=1, \ldots, N .
$$

Следовательно, для всех $N$ таких, что $c^{\prime} b_{N} \sqrt{n / N} \geqslant 1$, с вероятностью, большей $1-c_{1} \exp \left\{-c_{2} n A_{N}^{-1}+c_{3} N\right\}$,

$$
\begin{aligned}
& \sup _{f \in B_{N}} \mathbf{E}_{f}\left\{\|\hat{f}-f\|^{2} \mid X_{1}, \ldots, X_{n}\right\} \\
& \geqslant \frac{1}{2 \sqrt{2 \pi}} \int_{-1 / 2}^{1 / 2} u^{2} e^{-u^{2} / 2} d u \operatorname{tr}\left(Z_{N}^{*} D^{-1} Z_{N}\right)^{-1} \geqslant c_{0} \frac{N}{n} .
\end{aligned}
$$

Теорема доказана.

П р и м е р 4.1. Легко видеть, что в обоих примерах раздела 3 поперечники Бернштейна $b_{N} \geqslant c \sqrt{L} d^{\beta} N^{-\beta}$, где $c$ - положительная постоянная. Чтобы в этом убедиться, достаточно рассмотреть совокупность $B_{N}$ всех функций

$$
f(x)=\sqrt{2} \sum_{k=1}^{d} \sum_{j=1}^{T}\left(a_{j k} \sin \left(2 \pi j x_{k}\right)+\cos \left(2 \pi j x_{k}\right)\right),
$$

для которых $T \sim N d^{-1}$, а

$$
\|f\|^{2}=\sum_{k} \sum_{j}\left(\left|a_{j k}\right|^{2}+\left|b_{j k}\right|^{2}\right) \leqslant L T^{-2 \beta} .
$$

Все функции $f \in \mathbf{F}$, ибо для них $\sum_{k} \sum_{j}\left(\left|a_{j k}\right|^{2}+\left|b_{j k}\right|^{2}\right)|j|^{2 \beta} \leqslant L$. Радиус $N$-мерного диска $B_{N}$ не меньше, чем $c \sqrt{L}\left(d N^{-1}\right)^{\beta}$. В силу теоремы 4.1 
для любых оценок $\hat{f}$ функции $f$

$$
\begin{aligned}
\mathbf{P} & \left\{\sup _{f \in \mathbf{F}} \mathbf{E}_{f}\left\{\|f-\hat{f}\|^{2} \mid X_{1}, \ldots, X_{n}\right\} \geqslant C\left(\frac{d}{n}\right)^{2 \beta /(2 \beta+1)}\right\} \\
& \geqslant 1-\exp \left\{-c\left[\left(\frac{n}{d}\right)^{2 \beta /(2 \beta+1)}-n^{-1 /(2 \beta+1)} d^{2 \beta /(2 \beta+1)}\right]\right\} .
\end{aligned}
$$

\section{5. Границы точности оценивания и $\varepsilon$-энтропия.}

5.1. Здесь мы укажем границы точности оценивания $f$, выраженные в терминах $\varepsilon$-энтропии множества F. Энтропийные характеристики подмножеств метрических пространств были введены А. Н. Колмогоровым в 1956 году в работе [11], см. также [12], [17], [2]. Нам будет удобнее иметь дело не столько с $\varepsilon$-энтропией, сколько с эквивалентной ей характеристикой, $\varepsilon$-емкостью множества $\mathbf{F}$.

Пусть $N_{\varepsilon}=N_{\varepsilon}(\mathbf{F})$ означает максимально возможное число $\varepsilon$-различимых в метрике $L_{2}(\mathfrak{X})$ элементов $f_{1}, \ldots, f_{N}$ множества $\mathbf{F}$, т.е. таких точек $f_{i} \in \mathbf{F}$, что $\left\|f_{i}-f_{j}\right\|>\varepsilon$ для всех $i \neq j$. Величина $C_{\varepsilon}(\mathbf{F})=\ln N_{\varepsilon}(\mathbf{F})$ называется $\varepsilon$-емкостью множества $\mathbf{F}$.

Ниже для простоты мы, как и в предыдущем разделе, считаем, что при фиксированных $X_{1}, \ldots, X_{n}$ все остатки $\xi_{i}=Y_{i}-f\left(X_{i}\right)$ имеют гауссовские распределения со средними нуль и дисперсиями $r^{2}\left(X_{i}\right)$.

Кроме того, тоже для простоты, мы считаем ниже, что

$$
\sup _{f \in \mathbf{F}} \sup _{x}|f(x)|=A<\infty \text {. }
$$

Теорема 5.1. Для любого $\varepsilon>1 / \sqrt{n}$ найдется оченка $\hat{f}_{\varepsilon}$ функиии регрессии $f$ такая, что для всех $r>0$

$$
\begin{aligned}
& \mathbf{P}\left\{\mathbf{E}\left\{\left\|f-\hat{f}_{\varepsilon}\right\|^{r} \mid X_{1}, \ldots, X_{n}\right\} \geqslant \varepsilon^{r}+c_{r} \exp \left\{-\frac{n \varepsilon}{\sigma^{2}}+C_{\varepsilon}(\mathbf{F})\right\}\right. \\
& \leqslant C_{r} \exp \left\{-\frac{c n \varepsilon^{2}}{A^{4} \sigma^{2}}+C_{\varepsilon}(\mathbf{F})\right\} .
\end{aligned}
$$

Д о к а з а т ел ь с т в о. Пусть $\left\{f_{1}, \ldots, f_{N}\right\}-$ какое-нибудь максимальное $\varepsilon$-различимое подмножество множества $\mathbf{F}$, так что $N=N_{\varepsilon}(\mathbf{F})$. Рассмотрим статистику

$$
I\left(f_{j}\right)=\sum_{i=1}^{n}\left(Y_{i}-f_{j}\left(X_{i}\right)\right)^{2}
$$

и примем в качестве оценки $\hat{f}(x)$ ту из функций $f_{1}, \ldots, f_{N}$, которая доставляет минимум функционалу (5.2) (если точек минимума несколько, мы выбираем в качестве $\hat{f}$ любую из этих точек). Пусть $f_{a}$ - ближайшая к $f$ точка из числа $\left\{f_{1}, \ldots, f_{N}\right\}$. Тогда $\left\|f-f_{a}\right\| \leqslant \varepsilon$. Поэтому событие 
$\{\|f-\hat{f}\|>k \varepsilon\}$ влечет событие $\left\{\left\|f-f_{a}\right\|>(k-1) \varepsilon\right\}$. Следовательно, $\mathbf{P}\left\{\|f-\hat{f}\|>k \varepsilon \mid X_{1}, \ldots, X_{n}\right\} \leqslant \mathbf{P}\left\{\|f-\hat{f}\|>(k-1) \varepsilon \mid X_{1}, \ldots, X_{n}\right\}$

$$
\begin{aligned}
& \leqslant \sum_{j:\left\|f_{j}-f_{a}\right\|>(k-1) \varepsilon} \mathbf{P}\left\{\sum_{i}\left(Y_{i}-f_{a}\left(X_{i}\right)\right)^{2}>\sum_{i}\left(Y_{i}-f_{j}\left(X_{i}\right)\right)^{2} \mid X_{1}, \ldots, X_{n}\right\} \\
& =\sum_{j:\left\|f_{j}-f_{a}\right\|>k \varepsilon} \mathbf{P}\left\{2 \sum_{i=1}^{n} \xi_{i}\left(f\left(X_{i}\right)-f_{a}\left(X_{i}\right)\right)+\sum_{i=1}^{n}\left(f\left(X_{i}\right)-f_{a}\left(X_{i}\right)\right)^{2}\right. \\
& \left.\quad \geqslant 2 \sum_{i=1}^{n} \xi_{i}\left(f\left(X_{i}\right)-f_{a}\left(X_{i}\right)\right)+\sum_{i=1}^{n}\left(f\left(X_{i}\right)-f_{j}\left(X_{i}\right)\right)^{2} \mid X_{1}, \ldots, X_{n}\right\} \\
& =\sum_{j:\left\|f_{j}-f_{a}\right\|>(k-1) \varepsilon} \mathbf{P}\left\{2 \sum_{i=1}^{n} \xi_{i}\left(f\left(X_{i}\right)-f_{a}\left(X_{i}\right)\right)\right. \\
& \left.\geqslant \sum_{i=1}^{n}\left(f\left(X_{i}\right)-f_{j}\left(X_{i}\right)\right)^{2}-\sum_{i=1}^{n}\left(f\left(X_{i}\right)-f_{a}\left(X_{i}\right)\right)^{2} \mid X_{1}, \ldots, X_{n}\right\} .
\end{aligned}
$$

Положим $\eta_{i}=\left[f\left(X_{i}\right)-f_{j}\left(X_{i}\right)\right]^{2}$. Величины $\eta_{i}$ независимы, $\left|\eta_{i}\right| \leqslant 4 A^{2}$, a $\mathbf{E} \eta_{i} \geqslant(k-2) \varepsilon$. Поэтому, полагая $\zeta_{i}=\eta_{i}-\mathbf{E} \eta_{i}$, найдем, что

$$
\begin{aligned}
& \mathbf{P}\left\{\sum_{i=1}^{n} \eta_{i}<2^{-1}(k-2) n \varepsilon\right\} \leqslant \exp \left\{-2^{-1} t(k-2) n \varepsilon\right\} \cdot \prod_{1}^{n} \mathbf{E} e^{t \zeta_{i}} \\
& \quad \leqslant \exp \left\{-2^{-1} t(k-2) n \varepsilon\right\} \cdot\left(1+\sum_{r=2}^{\infty} \frac{t^{r}}{r !} \mathbf{E}\left|\zeta_{i}\right|^{r}\right)^{n} \\
& \quad \leqslant \exp \left\{-2^{-1} t(k-2) n \varepsilon\right\} \cdot\left(1+4 t^{2} A^{4} e^{4 t A^{2}}\right)^{n} \\
& \quad \leqslant \exp \left\{-2^{-1} t(k-2) \varepsilon+4 n t^{2} A^{4} e^{4 t A^{2}}\right\} .
\end{aligned}
$$

Выбирая здесь $t=c_{0} k \varepsilon A^{-4}$, где $c_{0}$ - подходящая положительная постоянная, приходим к неравенству

$$
\mathbf{P}\left\{\sum_{i=1}^{n} \eta_{i}<2^{-1}(k-2) n \varepsilon\right\} \leqslant \exp \left\{-c_{1} n k^{2} \varepsilon^{2} A^{-4}\right\} .
$$

Аналогично и

$$
\mathbf{P}\left\{\sum_{i=1}^{n}\left(f\left(X_{i}\right)-f_{a}\left(X_{i}\right)\right)^{2}>\frac{k n \varepsilon}{4}\right\} \leqslant \exp \left\{-c_{2} n k^{2} \varepsilon^{2} A^{-4}\right\} .
$$

При фиксированных $X_{1}, \ldots, X_{n}$ суммы $2 \sum_{i=1}^{n} \xi_{i}\left(f\left(X_{i}\right)-f_{a}\left(X_{i}\right)\right)$ имеют нормальное распределение со средним нуль и дисперсией

$$
4 \sum_{i=1}^{n} r^{2}\left(X_{i}\right)\left(f\left(X_{i}\right)-f_{a}\left(X_{i}\right)\right)^{2} \leqslant 4 \sigma^{2} \sum_{i=1}^{n}\left(f\left(X_{i}\right)-f_{a}\left(X_{i}\right)\right)^{2} .
$$

Поэтому для $k \geqslant 5$ с вероятностью, большей

$$
1-\exp \left\{-c_{3} n k^{2} \varepsilon^{2} A^{-4}\right\} \cdot N=1-\exp \left\{-c_{3} n k^{2} \varepsilon^{2} A^{-4}+C_{\varepsilon}(\mathbf{F})\right\},
$$


выполняется неравенство

$$
\mathbf{P}\left\{\|f-\hat{f}\| \geqslant k \varepsilon \mid X_{1}, \ldots, X_{n}\right\} \leqslant c_{4} N \exp \left\{-c_{5} k \varepsilon n \sigma^{-2}\right\} .
$$

Из этого неравенства в свою очередь следует, что с вероятностью, большей $1-C_{1}(r) \exp \left\{-c_{6} n \varepsilon^{2} n \varepsilon^{2} A^{-4}\right\} \cdot N$,

$$
\mathbf{E}\left\{\|f-\hat{f}\|^{r} \mid X_{1}, \ldots, X_{n}\right\} \leqslant \varepsilon^{r}+N C_{2}(r) \exp \left\{-c_{5} \varepsilon n \sigma^{-2}\right\} .
$$

Теорема доказана.

Следствие 5.1. Пусть $C_{\varepsilon}(\mathbf{F}) \uparrow \infty$, когда $\varepsilon \rightarrow 0$. Обозначим $\kappa_{n}$ корень уравнения $c_{6} n \varepsilon^{2} A^{-4}=2 C_{\varepsilon}(\mathbf{F})$. Тогда

$$
\mathbf{P}\left\{\mathbf{E}\left\{\left\|f-\hat{f}_{n}\right\|^{r} \mid X_{1}, \ldots, X_{n}\right\} \geqslant \kappa_{n}^{r}\right\} \leqslant C \exp \left\{-c n \kappa_{n}^{2} A^{-4}\right\} .
$$

Постоянные $C, c$ могут зависеть от $M, m, \sigma, \sigma_{0}, r$.

3 а м е ч а н и е. Поскольку $\varepsilon$-емкость $C_{\varepsilon}^{*}(\mathbf{F})$ и $\varepsilon$-энтропия $H_{\varepsilon}(\mathbf{F})$ множества $\mathbf{F}$ связаны соотношениями (см. [12])

$$
C_{2 \varepsilon}(\mathbf{F}) \leqslant H_{\varepsilon}(\mathbf{F}) \leqslant C_{\varepsilon}(\mathbf{F}),
$$

то неравенства теоремы 5.1 и следствия 5.1 нетрудно переписать в терминах $\varepsilon$-энтропии.

5.2. Пример. Предположим, как и в разделе 3 , что $\mathfrak{X}$ - это единичный куб $[0,1]^{d}$ пространства $\mathbf{R}^{d}$. Предположим далее, что $\mathbf{F}-$ это класс функций $f(x), x=\left(x_{1}, \ldots, x_{d}\right)$, представимых в виде

$$
f(x)=g\left(f_{1}\left(x_{1}\right), \ldots, f_{d}\left(x_{d}\right)\right)
$$

где $g$ - известная функция, удовлетворяющая условию Липшица, а функции $f_{j} \in F_{j}$, где $F_{j}$ обозначает класс функций, определенных на $[0,1]$ и удовлетворяющих там условию (3.1) с $\beta>\frac{1}{2}$.

А.Н. Колмогоров показал (см. [12], [2]), что при $\varepsilon \rightarrow 0 \varepsilon$-емкости $C_{\varepsilon}\left(F_{j}\right) \asymp(1 / \varepsilon)^{1 / \beta}$. Поэтому

$$
C_{\varepsilon}(\mathbf{F}) \leqslant c d\left(\frac{1}{\varepsilon}\right)^{1 / \beta}
$$

Отсюда и из (5.7) следует, что в условиях примера найдется оценка $\hat{f}_{n}$ функции $f$ такая, что

$$
\begin{aligned}
& \mathbf{P}\left\{\sup _{f} \mathbf{E}_{f}\left\{\left\|f-\hat{f}_{n}\right\|^{2} \mid X_{1}, \ldots, X_{n}\right\}>\left(\frac{d}{n}\right)^{2 \beta /(2 \beta+1)}\right\} \\
& \quad \leqslant C \exp \left\{-c\left(n d^{-1}\right)^{1 / 2 \beta+1}\right\},
\end{aligned}
$$

причем константы $c, C$ зависят от $m, M, \sigma, \sigma_{0}, A, \beta, L$, постоянной Липшица функции $g$, но не от размерности $d$.

Ясно, что модель аддитивной регрессии есть весьма частный случай моделей вида (5.8). Этот последний класс можно еще расширить, 
допуская, например, что функция $g$ тоже неизвестна, но принадлежит известному классу $G$ функций $g$, удовлетворяющих условию Липшица с общей константой $L$ :

$$
|g(x)-g(y)| \leqslant L|x-y|
$$

В этом случае, как легко видеть,

$$
H_{\varepsilon(1+L \sqrt{d})}(\mathbf{F}) \leqslant H_{\varepsilon}(G)+\sum_{1}^{d} H_{\varepsilon}\left(F_{j}\right) .
$$

\section{СПИСОК ЛИТЕРАТУРЫ}

1. Ахиезер Н. И. Лекции по теории аппроксимации. М.: Наука, 1965, 407 с.

2. Бабенко К. И. (ред.). Теоретические основы и конструирование численных алгоритмов задач математической физики. М.: Наука, 1979, 295 с.

3. Horowitz J. L. Semiparametric Methods in Econometrics. Berlin: Springer-Verlag, 1998, 204 p. (Lecture Notes in Statist., v. 131.)

4. Зигмунд А. Тригонометрические ряды. Т. І. М.: Мир, 1965, 615 с.

5. Ибрагимов И. А. Об одной задаче оценивания функции регрессии. - Докл. РАН, 2001, т. 381, № 6, с. 1-4.

6. Ибрагимов И.А., Хасьминский Р. З. Непараметрические оценки регрессии. Докл. АН СССР, 1980, т. 252, № 4, с. 780-784.

7. Ибрагимов И. А., Хасьминский Р. З. О границах качества непараметрического оценивания регрессии. - Теория вероятн. и ее примен., 1982, т. 27, в. 1, с. 81-94.

8. Ибрагимов И. А., Хасьминский Р. З. Асимптотические границы качества непараметрического оценивания регрессии в $L_{p}$. - Зап. научн. семинаров ЛОМИ, 1980 , т. 97, c. $88-101$.

9. Ibragimov I., Khasminskii R. On density estimation in the view of Kolmogorov's ideas in approximation theory. - Ann. Statist., 1990, v. 18, № 3, p. 999-1010.

10. Kolmogoroff $A$. Über die beste Annäherung von Funktionen einer gegebenen Funktionenklasse. - Ann. Math., 1936, v. 37, p. 107-110. Рус. пер. в кн.: Колмогоров А. H. Избранные труды. Математика и механика. М.: Наука, 1985, с. 186-189.

11. Колмогоров A.H. О некоторых асимптотических характеристиках вполне ограниченных метрических пространств. - Докл. АН СССР, 1956, т. 108, № 3, с. 385388.

12. Колмогоров $A . H$., Тихомиров B. M. $\varepsilon$-энтропия и $\varepsilon$-емкость множеств в функциональных пространствах. - Успехи матем. наук, 1959, т. 14, № 2, с. 3-86. См. также: Колмогоров $A . H$. Теория информации и теория алгоритмов. М.: Наука, 1987 , c. $119-192$.

13. Никольский С. М. Приближение функций многих переменных и теоремы вложения. М.: Наука, 1969, 480 с.

14. Stone Ch. Optimal global rates of convergence for nonparametric regression. - Ann. Statist., 1982, v. 10, № 4, p. 1040-1053.

15. Stone Ch. Additive regression and other nonparametric models. - Ann. Statist., 1985 , v. 13 , № 2 , p. 689-705.

16. Тихомиров В. М. Некоторые вопросы теории приближений. М.: Изд-во Моск. унта, 1976, $304 \mathrm{c.}$

17. Тихомиров В. М. Теория приближения. - Итоги науки и техники. Совр. проблемы матем. Фундам. направления. Т. 14. М.: ВИНИТИ, 1987, с. 103-260.

18. Ченцов H. H. Оценка неизвестной плотности распределения по наблюдениям. Докл. АН СССР, 1962, т. 147, № 1, с. 45-48. 